\begin{tabular}{|c|l|}
\hline Title & Skyrmion in spinor condensates and its stability in trap potentials \\
\hline Author(s) & Tokuno, A.; Mitamura, Y.; Oshikawa, M.; Herbut, I. F. \\
\hline Citation & $\begin{array}{l}\text { Physical Review A, 79(5), 053626 } \\
\text { https://doi.org/10.1103/PhysRevA.79.053626 }\end{array}$ \\
\hline Issue Date & 2009-05 \\
\hline Doc URL & http://hdl.handle.net/2115/38745 \\
\hline Rights & ○ 2009 The A merican Physical Society \\
\hline Type & article \\
\hline File Information & 79-5_053626.pdf \\
\hline
\end{tabular}

Instructions for use 


\title{
Skyrmion in spinor condensates and its stability in trap potentials
}

\author{
A. Tokuno, ${ }^{1}$ Y. Mitamura, ${ }^{2,3}$ M. Oshikawa, ${ }^{2}$ and I. F. Herbut ${ }^{4}$ \\ ${ }^{1}$ Department of Applied Physics, Hokkaido University, Sapporo 060-8628, Japan \\ ${ }^{2}$ Institute for Solid State Physics, University of Tokyo, Kashiwa 227-8581, Japan \\ ${ }^{3}$ Department of Physics, Tokyo Institute of Technology, Oh-okayama, Meguro-ku, Tokyo 152-8551, Japan \\ ${ }^{4}$ Department of Physics, Simon Fraser University, Burnaby, British Columbia, Canada V5A 1S6
}

(Received 15 December 2008; published 15 May 2009)

\begin{abstract}
A necessary condition for the existence of a skyrmion in two-component Bose-Einstein condensates with SU(2) symmetry was recently provided by two of the authors [I. F. Herbut and M. Oshikawa, Phys. Rev. Lett. 97, 080403 (2006)], by mapping the problem to a classical particle in a potential subject to time-dependent dissipation. Here we further elaborate this approach. For two classes of models, we demonstrate the existence of the critical dissipation strength above which the skyrmion solution does not exist. Furthermore, we discuss the local stability of the skyrmion solution by considering the second-order variation. A sufficient condition for the local stability is given in terms of the ground-state energy of a one-dimensional quantum-mechanical Hamiltonian. This condition requires a minimum number of bosons, for a certain class of the trap potential. In the optimal case, the minimum number of bosons can be as small $\sim 10^{4}$.
\end{abstract}

DOI: 10.1103/PhysRevA.79.053626

PACS number(s): 03.75.Hh, 05.30.Jp, 03.75.Mn, 11.27.+d

\section{INTRODUCTION}

Topological defects often play a fundamental role in our understanding of phases of matter and the transitions between them. The best understood examples are probably vortices and vortex loops in superfluids and $\mathrm{O}(2)$ magnets in two and three dimensions, which are responsible for the very existence of the high-temperature phase, and completely determine the universality class of the phase transition [1]. The next in order of complexity are the topological defects in the $\mathrm{O}(3)$-symmetric Heisenberg model, which allows skyrmions in two dimensions [2] and hedgehog-like configurations (a point defect around which spins point outward) in three dimensions [3]. While the former only renormalize the coupling constant, the role of the latter is less clear [4]. All of the above, however, represent configurations topologically distinct from vacuum, which provides them with local stability.

In this paper, we study the stability of the topologically nontrivial skyrmion configuration in theories with $\mathrm{O}(4)$ symmetry. Such a symmetry arises in complex condensates with an internal spin-1/2-like quantum number [5], for example. Realizations of such spinor condensates are found in models of inflatory cosmology [6], Bose-Einstein condensation of ${ }^{87} \mathrm{Rb}[7]$, and bosonic ferromagnetism $[5,8]$, and in effective theories of high-temperature superconductivity $[9,10]$ and of deconfined criticality [11]. The Higgs sector of the Weinberg-Salam model of electroweak interactions represents another closely related example, with a spinor condensate coupled to gauge fields. In our problem in three dimensions, there exists a topologically nontrivial mapping to the order-parameter space, thanks to the fact that the third homotopy group of $S^{3}$ is the group of integers. However, the topology alone turns out to be insufficient to guarantee local stability of the skyrmion. This may be understood already in terms of the classic Derick theorem [12]. Recently, a more general proof that the skyrmion cannot be a stationary point of the action for the spinor Bose-Einstein condensate (BEC) in free space was given by two of the present authors [13], based on the analogy between the Euler-Lagrange equations and the classical mechanics of a particle in a time-dependent dissipative environment [14]. The advantage of this alternative point of view at the old problem is that it provides one with a simple way of constructing the external potentials which would indeed lead to skyrmion as the solution of the Euler-Lagrange equations. In Ref. [13], three such special potentials were presented. In this paper, we further develop this approach.

First we analyze the construction of the solution based on the ansatz proposed in Ref. [13]. There, a time-dependent dissipation which is odd in time was introduced, to allow an odd solution. However, we find that the symmetry argument does not always work, and there is a critical dissipation strength above which the odd solution no longer exists even for an odd dissipation. Next, we study the stability of the skyrmion in a generalized class of the potentials introduced in [13] with respect to small variations. We map the problem to an effective quantum-mechanical eigenvalue problem and determine the region of local stability in the parameter space. A particularly interesting result of our analysis is that the stable skyrmion requires a minimal number of particles in the trap, estimated here to be $\sim 10^{4}$ in the optimal case.

The paper is organized as follows. In Sec. II, we present basic formulation of the problem. A classical equation of motion with time-dependent dissipation determines the skyrmion solution. On the other hand, a quantum-mechanical eigenvalue problem determines the local stability of the solution. In Sec. III, we discuss the construction of the skyrmion solution based on the odd-function ansatz. We determine the critical dissipation strength, which separates the region with and without a skyrmion solution. In Sec. IV, we present the numerical solution of the equation of motion for a few cases. The existence of the critical dissipation strength, as well as related theoretical predictions, is confirmed numerically. Furthermore, the local stability of the obtained solution is also analyzed by solving the quantum-mechanical eigenvalue problem. The minimal number of bosons required to satisfy the sufficient condition for the local stability is numerically 
obtained as a function of parameter. Section $\mathrm{V}$ is devoted to summary of the paper.

\section{GENERAL DISCUSSION ON THE SKYRMION SOLUTION}

\section{A. Basic equations}

We begin by reviewing the derivation of the skyrmion solution for the two-component (spinor) BEC in an external potential, formulated previously in Ref. [13]. The derivation is based on the Euler-Lagrange equations, and leads to the necessary condition for the existence of the skyrmion solution. We will then proceed to examine the stability of the skyrmion by taking into account the second-order variation.

Let us consider the two-component bosons in threedimensional continuum space in the external confinement potential $V(\boldsymbol{r})$. The system can be described by the following effective action via the path-integral formalism [1]:

$$
\begin{gathered}
S=\int_{0}^{\beta} d \tau \int d^{3} \boldsymbol{r} \mathcal{L}, \\
\mathcal{L}=\Phi^{\dagger}\left(\partial_{\tau}-\frac{\hbar^{2}}{2 m} \nabla^{2}-\mu+V(\boldsymbol{r})\right) \Phi+\frac{U}{2}\left(\Phi^{\dagger} \Phi\right)^{2},
\end{gathered}
$$

where $\Phi^{\dagger}(\tau, \boldsymbol{r})=\left(\Phi_{1}^{*}(\tau, \boldsymbol{r}), \Phi_{2}^{*}(\tau, \boldsymbol{r})\right)$ is a two-component bosonic field with the mass $m$, which satisfies the periodic boundary condition $\Phi(0, r)=\Phi(\beta, r)$, where $\beta=1 / k_{\mathrm{B}} T$. The bosons interact with each other by the repulsive contact interaction $U>0 . \mu$ is the chemical potential. In addition to the usual $\mathrm{U}(1)$ symmetry corresponding to the conservation of the number of the bosons, the system is invariant with respect to the global $\mathrm{SU}(2)$ transformation of the boson field, $\Phi \rightarrow \Phi^{\prime}(\tau, \boldsymbol{r})=\mathcal{U} \Phi(\tau, \boldsymbol{r})$, where $\mathcal{U}$ is an $\operatorname{SU}(2)$ matrix. As usual, the trap potential $V(\boldsymbol{r})$ has been included in the definition of the action or the corresponding Hamiltonian.

Next, we introduce the dimensionless parameters and fields by rescaling:

$$
\begin{aligned}
\Psi(\widetilde{\tau}, \widetilde{\boldsymbol{r}}) & =\xi^{3 / 2} \Phi\left(\mu \tau, \xi^{-1} \boldsymbol{r}\right), \\
\tilde{V}(\widetilde{\boldsymbol{r}}) & =\mu^{-1} V\left(\xi^{-1} \boldsymbol{r}\right), \\
\tilde{U} & =\mu^{-1} \xi^{-3} U,
\end{aligned}
$$

where $\xi=\hbar / \sqrt{m \mu}$ is the constant length scale. Effective action (1) may be written now in terms of the dimensionless parameters as

$$
\begin{gathered}
S=\int_{0}^{\mu \beta} d \tilde{\tau} \int d^{3} \widetilde{\boldsymbol{r}} \mathcal{L}, \\
\mathcal{L}=\Psi^{\dagger}\left[\partial_{\tilde{\tau}}-\frac{1}{2} \widetilde{\nabla}^{2}-1+\tilde{V}(\widetilde{\boldsymbol{r}})\right] \Psi+\frac{\tilde{U}}{2}\left(\Psi^{\dagger} \Psi\right)^{2} .
\end{gathered}
$$

Let us focus on classical field configurations independent of the imaginary time. It will prove convenient to represent the $\tau$-independent field $\Psi_{c}(\widetilde{\boldsymbol{r}})$ by an amplitude $f(\widetilde{\boldsymbol{r}})$ and a two-component complex spinor configuration $a(\widetilde{\boldsymbol{r}}): \Psi_{c}(\widetilde{\boldsymbol{r}})$ $=f(\widetilde{\boldsymbol{r}}) a(\widetilde{\boldsymbol{r}})$. The spinor $a(\widetilde{\boldsymbol{r}})$ is normalized as $a^{\dagger}(\widetilde{\boldsymbol{r}}) a(\widetilde{\boldsymbol{r}})=1$. In terms of $f$ and $a$, we can rewrite the effective action for the $\tau$-independent field configuration $\Psi_{c}$,

$$
\begin{gathered}
S_{c}=\mu \beta \int d^{3} \widetilde{\boldsymbol{r}} \mathcal{L}_{c}, \\
\mathcal{L}_{c}=\frac{1}{2}(\widetilde{\nabla} f)^{2}+\frac{1}{2} f^{2} \widetilde{\nabla} a^{\dagger} \cdot \tilde{\nabla} a+(\tilde{V}-1) f^{2}+\frac{\tilde{U}}{2} f^{4},
\end{gathered}
$$

where we have used the relation $\tilde{\nabla}\left(a^{\dagger} a\right)=\left(\widetilde{\nabla} a^{\dagger}\right) a+a^{\dagger}(\tilde{\nabla} a)$ $=0$ deduced from the normalization condition for the spinor $a(\tilde{\boldsymbol{r}})$. The stationary state also needs to satisfy the boundary condition

$$
\lim _{|\widetilde{\boldsymbol{r}}| \rightarrow \infty}|\widetilde{\boldsymbol{r}}|^{2} f(\widetilde{\boldsymbol{r}})^{2} \tilde{\nabla} a(\widetilde{\boldsymbol{r}})=0 .
$$

It guarantees the stability of the solution with respect to small rotations of the spinor $a(\tilde{\boldsymbol{r}})$ at the infinitely remote boundary of the system.

Let us take the variation in action (6) around the classical field $\Psi_{c}(\boldsymbol{r})$ using the following expressions for the amplitude and the spinor configuration:

$$
\begin{aligned}
& f(\widetilde{\boldsymbol{r}})=f_{0}(\widetilde{\boldsymbol{r}})+\delta f(\widetilde{\boldsymbol{r}}), \\
& a(\widetilde{\boldsymbol{r}})=a_{0}(\widetilde{\boldsymbol{r}})+\delta a(\widetilde{\boldsymbol{r}}),
\end{aligned}
$$

where $\delta f(\widetilde{\boldsymbol{r}})$ and $\delta a(\widetilde{\boldsymbol{r}})$ are the variations around $\Psi_{c}(\tilde{\boldsymbol{r}})$ for the density profile and the spinor configuration, respectively. $f_{0}(\widetilde{\boldsymbol{r}})$ and $a_{0}(\widetilde{\boldsymbol{r}})$ are the amplitude and the spinor of some stationary field configuration. In particular, $a_{0}(\widetilde{\boldsymbol{r}})$ will assume a form corresponding to the skyrmion solution, which is to be defined shortly. Substituting into the action, action (6) can be written as

$$
S_{c}=\mu \beta \int d^{3} \widetilde{\boldsymbol{r}}\left[\mathcal{L}_{0}+\mathcal{L}_{1}+\mathcal{L}_{2}+\cdots\right]
$$

where $\mathcal{L}_{i}$ is the Lagrangian density related to the $i$ th order of the variation $\delta f(\widetilde{\boldsymbol{r}})$ and $\delta a(\widetilde{\boldsymbol{r}})$. The Lagrangian densities up to the second-order variation are then expressed as

$$
\mathcal{L}_{0}=\frac{1}{2}\left(\tilde{\nabla} f_{0}\right)^{2}+\frac{1}{2} f_{0}^{2} \tilde{\nabla} a_{0}^{\dagger} \cdot \tilde{\nabla} a_{0}+(\tilde{V}-1) f_{0}^{2}+\frac{\tilde{U}}{2} f_{0}^{4},
$$

$$
\begin{aligned}
\mathcal{L}_{1}= & {\left[-\tilde{\nabla}^{2} f_{0}+\left\{\tilde{\nabla} a_{0}^{\dagger} \cdot \tilde{\nabla} a_{0}+2 \tilde{V}-2\right\} f_{0}+2 \tilde{U} f_{0}^{3}\right] \delta f } \\
& -\frac{1}{2}\left[\tilde{\nabla} \cdot\left(f_{0}^{2} \widetilde{\nabla} a_{0}^{\dagger}\right) \delta a+\text { H.c. }\right], \\
\mathcal{L}_{2}= & \delta f\left[-\frac{1}{2} \widetilde{\nabla}^{2}+\tilde{V}-1+3 \tilde{U} f_{0}^{2}-\frac{3}{2} \widetilde{\nabla} a_{0}^{\dagger} \cdot \widetilde{\nabla} a_{0}\right] \delta f \\
+ & \frac{1}{2}\left[f_{0}\left(\tilde{\nabla} \delta a^{\dagger}\right)+2\left(\tilde{\nabla} a_{0}^{\dagger}\right) \delta f\right] \cdot\left[f_{0}(\tilde{\nabla} \delta a)+2\left(\tilde{\nabla} a_{0}\right) \delta f\right] .
\end{aligned}
$$




\section{B. Mapping to a problem in classical mechanics}

Setting $\int d^{3} \widetilde{\boldsymbol{r}} \mathcal{L}_{1}=0$ for any $\delta f(\widetilde{\boldsymbol{r}})$ and $\delta a(\widetilde{\boldsymbol{r}})$ leads to the Euler-Lagrange equations giving the extremum of the action:

$$
\begin{gathered}
-\tilde{\nabla}^{2} f_{0}+2\left[\frac{1}{2} \tilde{\nabla} a_{0}^{\dagger} \cdot \tilde{\nabla} a_{0}+\tilde{V}-1\right] f_{0}+2 \tilde{U} f_{0}^{3}=0, \\
2 \tilde{\nabla} \cdot\left(f_{0}^{2} \tilde{\nabla} a_{0}\right)=\left[a_{0}^{\dagger} \tilde{\nabla} \cdot\left(f_{0}^{2} \tilde{\nabla} a_{0}\right)+\left\{\tilde{\nabla} \cdot\left(f_{0}^{2} \tilde{\nabla} a_{0}^{\dagger}\right)\right\} a_{0}\right] a_{0} .
\end{gathered}
$$

The latter equation follows by recalling that $a^{\dagger} a=1$, so that $a_{0}^{\dagger} \delta a+\delta a^{\dagger} a_{0}=0$.

For simplicity, we further assume that the external potential is spherically symmetric, $\tilde{V}(\widetilde{\boldsymbol{r}})=\tilde{V}(\widetilde{r})$, where $\tilde{r}=|\widetilde{\boldsymbol{r}}|$. Then, in terms of the spinor configuration, we can adopt the most general ansatz with the same spherical symmetry [3],

$$
\begin{gathered}
f_{0}(\widetilde{\boldsymbol{r}})=f_{0}(\widetilde{r}), \\
a_{0}(\widetilde{\boldsymbol{r}})=\left(\begin{array}{c}
\sin \omega(\widetilde{r}) \cos \theta-i \cos \omega(\widetilde{r}) \\
\sin \omega(\widetilde{r}) \sin \theta e^{-i \phi}
\end{array}\right),
\end{gathered}
$$

where $\tilde{\boldsymbol{r}}=(\widetilde{r} \sin \theta \cos \phi, \tilde{r} \sin \theta \sin \phi, \widetilde{r} \cos \theta)$. At the infinitely remote boundary, we impose $\omega(\tilde{r}=\infty)=N \pi$, where $N$ is any integer. If we additionally adopt the boundary condition $\omega(\widetilde{r}=0)=0$, and $\omega(\widetilde{r})$ changes from 0 to $N \pi$ as $\tilde{r}$ changes from 0 to infinity, ansatz (14) means that the spinor configurations wraps the three-dimensional sphere $S^{3} N$ times, and thus corresponds to the skyrmion solution. Hereafter, we restrict the discussion to the simplest case of $N=1$.

Let us then consider Euler-Lagrange equations (11) and (12) for $N=1$ skyrmions (13) and (14). Substituting Eqs. (13) and (14) into Euler-Lagrange equations (11) and (12), we obtain the following differential equations:

$$
\begin{aligned}
& -\frac{d^{2} f_{0}}{d \widetilde{r}^{2}}-\frac{2}{\widetilde{r}} \frac{d f_{0}}{d \tilde{r}}+2\left[\frac{1}{2}\left(\frac{d \omega}{d \widetilde{r}}\right)^{2}+\left(\frac{\sin \omega}{\tilde{r}}\right)^{2}+\tilde{V}-1\right] f_{0}+2 \widetilde{U} f_{0}^{3} \\
& =0 \\
& \frac{d^{2} \omega}{d \widetilde{r}^{2}}+\left[\frac{2}{\widetilde{r}}+\frac{2}{f_{0}} \frac{d f_{0}}{d \tilde{r}}\right] \frac{d \omega}{d \widetilde{r}}-\frac{\sin 2 \omega}{\widetilde{r}^{2}}=0
\end{aligned}
$$

To analyze these differential equations, it is very convenient to change the variable from $\tilde{r}$ to $t=\ln \tilde{r}$. Then, as $0<\widetilde{r}<\infty$, $-\infty<t<\infty$. Rewriting Eq. (16) in terms of $t$, we obtain

$$
\frac{d^{2} \bar{\omega}}{d t^{2}}=-\frac{d W}{d \bar{\omega}}-\eta(t) \frac{d \bar{\omega}}{d t},
$$

where $\bar{\omega}(t)=\omega\left(e^{t}\right)=\omega(\widetilde{r})$. This equation can be regarded as describing classical motion of a particle in an external potential with dissipation. $W(\bar{\omega})$ and $\eta(t)$, respectively, correspond to the potential energy and the "time"-dependent dissipation, given by

$$
W(\bar{\omega})=\frac{1}{2} \cos 2 \bar{\omega}
$$

$$
\eta(t)=1+\frac{d}{d t} \ln \vec{f}_{0}^{2}
$$

where $\bar{f}_{0}(t)=f_{0}\left(e^{t}\right)=f_{0}(\widetilde{r})$. In terms of the $N=1$ skyrmion solution, boundary conditions for $\omega(\widetilde{r})$ may be written as $\omega(0)=d \omega / d \widetilde{r}(0)=0$ at the origin of the three-dimensional space, and $\omega(\infty)=\pi, d \omega / d \tilde{r}=0$ at the infinitely remote boundary. These boundary conditions in terms of $t$ translate into

$$
\begin{gathered}
\bar{\omega}(-\infty)=0, \quad \frac{d \bar{\omega}}{d t}(-\infty)=0, \\
\bar{\omega}(\infty)=\pi, \quad \frac{d \bar{\omega}}{d t}(\infty)=0 .
\end{gathered}
$$

Integrating equation of motion (17) with respect to $t$, and imposing the set of boundary conditions (20), one obtains the following necessary condition for the existence of the skyrmion solution:

$$
\int_{-\infty}^{\infty} d t \eta(t)\left(\frac{d \bar{\omega}}{d t}\right)^{2}=0 .
$$

The condition implies that the total integrated dissipation in the problem vanishes [15]. It is now clear that a skyrmion solution exists only when the density profile takes a special form, so that the solution $\bar{\omega}(t)$ satisfies condition (21).

\section{Stability of the skyrmion and a quantum-mechanical eigenvalue problem}

The integral condition provides only the necessary condition for the existence of the skyrmion solution, but does not guarantee its stability. Here, we analyze the second-order variation, to obtain a further condition for the stable skyrmion solutions.

Let us assume that an appropriate external potential $\tilde{V}(\widetilde{r})$ is given so that the Euler-Lagrange equations allow a skyrmion solution $f_{0}(\widetilde{r})$ and $a_{0}(\widetilde{\boldsymbol{r}})$. For the obtained skyrmion solution to be stable against local variations, the secondorder variation in Lagrangian (10) has to be positive. Obviously, the second term in Eq. (10) is always positive. The positivity of the first term in Eq. (10) for arbitrary $\delta f(\widetilde{\boldsymbol{r}})$ and $\delta a(\widetilde{\boldsymbol{r}})$ is thus a sufficient condition for the local stability of the skyrmion. The first term being a quadratic form of $\delta f$, its positivity for an arbitrary $\delta f$ is equivalent to positive definiteness of the linear operator

$$
H=-\frac{1}{2} \widetilde{\nabla}^{2}+V_{\mathrm{eff}}(\widetilde{\boldsymbol{r}}),
$$

where

$$
V_{\mathrm{eff}}(\widetilde{\boldsymbol{r}})=\tilde{V}(\widetilde{r})-1+3 \tilde{U} f_{0}^{2}(\widetilde{r})-\frac{3}{2} \tilde{\nabla} a_{0}^{\dagger} \cdot \tilde{\nabla} a_{0} .
$$

Positive definiteness of $H$ means that all the eigenvalues of $H$ are positive. In fact, $H$ may be interpreted as the quantummechanical Hamiltonian for a single particle in the external potential $V_{\text {eff. }}$ The (sufficient) condition for the stability then corresponds to the ground-state energy of the Hamiltonian being positive. 
The external potential $\tilde{V}(\tilde{\boldsymbol{r}})$ depends on both the spinor part and the density profile of the skyrmion, as set by the Euler-Lagrange equations. According to Eq. (11), $V_{\text {eff }}(\widetilde{\boldsymbol{r}})$ is also spherically symmetric.

We should mention that even if the ground-state energy of Hamiltonian (22) is negative, it is possible that second-order variation (10c) is still positive, if the positive second term is large enough. We, however, will be unable to say more about this issue, and our discussion will be limited to the sufficient condition for the skyrmion stability formulated above.

\section{CONSTRUCTION OF SKYRMION SOLUTIONS FOR TRAPPED BECS}

\section{A. Odd-function ansatz}

Let us discuss a few concrete examples of skyrmion solutions. In a usual formulation, we seek a solution for a given trap potential $V(\boldsymbol{r})$. However, as discussed in Ref. [13], a generic trap potential does not allow a skyrmion solution. Thus, we solve the problem backward: first we determine the dissipation $\eta(t)$ so that equation of motion (17) has a solution $\bar{\omega}(t)$ which represents a skyrmion. The assumed dissipation determines the density profile of bosons. Finally, the trap potential $V(r)$ is determined so that it reproduces the chosen $\eta(t)$.

For convenience, here we introduce the new variable

$$
y \equiv \bar{\omega}-\frac{\pi}{2} .
$$

Equation of motion (17) is then rewritten as

$$
\frac{d^{2} y}{d t^{2}}=-\eta(t) \frac{d y}{d t}-\sin 2 y .
$$

Boundary conditions (20) read, in terms of $y$,

$$
\begin{gathered}
y(-\infty)=-\frac{\pi}{2}, \quad \frac{d y}{d t}(-\infty)=0, \\
y(\infty)=+\frac{\pi}{2}, \quad \frac{d y}{d t}(\infty)=0 .
\end{gathered}
$$

We observe that the boundary conditions at $t=+\infty$ would be automatically met if $y$ obeys boundary conditions (26) at $t$ $=-\infty$ and $y(t)$ is an odd function:

$$
y(t)=-y(-t) .
$$

This is sufficient to satisfy the original conditions (26) and (27), but not necessary. However, here we focus on finding the solutions $y(t)$ which are odd in $t$, because it is easier than solving the general problem.

Equation (25) is invariant under

$$
t \rightarrow-t, \quad y \rightarrow-y
$$

if $\eta(t)$ is also odd in $t$. Thus we choose an odd $\eta(t)$ so that we can find an odd solution $y(t)$. Although this is a somewhat restrictive choice, it is a useful ansatz in construction of skyrmion solutions.
However, it turns out that some choice of odd $\eta(t)$ actually does not allow an odd solution $y(t)$ which satisfies the boundary conditions at $t=-\infty$. Roughly speaking, if the dissipation is too strong, the particle which starts at $y=-\pi / 2$ with a vanishing speed at $t=-\infty$ cannot reach $y=0$ at $t=0$. We will examine two forms of odd $\eta(t)$ as examples:

$$
\begin{gathered}
\eta(t)=n[\theta(-t-1)-\theta(t-1)], \\
\eta(t)=-n \tanh t,
\end{gathered}
$$

where $n$ is a positive parameter. As we will demonstrate, for each case there is a critical parameter $n_{c}$; an appropriate odd solution exists only if $n<n_{c}$.

The existence of an odd solution can be discussed in the following manner. First we impose boundary conditions (26) only at $t=-\infty$. Of course, they do not completely fix a solution but allow a family of different solutions. This is evident by recalling that the trivial solution $y(t)=-\pi / 2$ satisfies Eq. (26).

Then we attempt to find, among the solutions, the one which satisfies $y(0)=0$. This would be the desired odd solution. The particle falls off the hill and approaches the potential minimum $y=0$. If the particle is still rolling down the hill $(-\pi / 2<y<0)$ when $t=0$, the particle is only accelerated by the negative friction for $t>0$ and it must eventually reach $y=0$ at some positive $t$. Namely, for any dissipation strength, there is a solution which satisfies $y(t)=0$ at a positive $t$. On the other hand, while $y<0$, the velocity $d y / d t$ should always be positive. Thus the smallest solution $t$ of $y(t)=0$ changes continuously. Therefore, if there is another solution which satisfies $y(t)=0$ at a negative $t$, there must be an odd solution with $y(0)=0$ thanks to the intermediate value theorem.

\section{B. Constant- $\eta$ regime}

In either case of Eq. (30) and (31), we observe that for $t$ $\ll-1$,

$$
\eta(t) \sim n .
$$

This simply represents the constant-dissipation coefficient. The equation of motion

$$
\frac{d^{2} y}{d t^{2}}=-n \frac{d y}{d t}-\sin 2 y
$$

in this regime is independent of time. Thus, for any solution $y(t)$, the translated solution $y(t+\tau)$ is also a solution for any $\tau$. Let us discuss the solutions of this equation. This will turn out to be useful in determining the critical parameter $n_{c}$ for the original equation of motion (25).

As we will see later, when $n \sim n_{c}$, the particle comes very close to the minimum $(|y| \ll 1)$ while still in the constant- $\eta$ regime $(t \ll-1)$. For small $y$, we may use the linearized equation of motion

$$
\frac{d^{2} y}{d t^{2}}=-n \frac{d y}{d t}-2 y,
$$

instead of the full nonlinear equation (33). Its solution can be easily obtained as 


$$
y(t)=A_{1} e^{-\lambda_{1}(t+\tau)}+A_{2} e^{-\lambda_{2}(t+\tau)},
$$

where

$$
\begin{aligned}
& \lambda_{1}=\frac{n-\sqrt{n^{2}-8}}{2}, \\
& \lambda_{2}=\frac{n+\sqrt{n^{2}-8}}{2} .
\end{aligned}
$$

One can choose an arbitrary large positive $\tau$, thanks to the translation invariance in time. On the other hand, taking a large negative $\tau$ (for a fixed $t$ ) makes $y$ large, and may invalidate linear approximation (34).

When $n<2 \sqrt{2}, \lambda_{1,2}$ are a complex conjugate pair and the solution represents a damped harmonic oscillation. In this case, there is obviously a solution which reaches $y=0$ within Eq. (33). Namely, there is a solution which satisfies $y(t)=0$ at a negative $t$. As we discussed in Sec. III A, the intermediate value theorem assures that there is an odd solution with $y(0)=0$ in this case. This means that $n_{c}>2 \sqrt{2}$.

Thus, in the following, we focus on $n>2 \sqrt{2}$. Then, the first term $\propto e^{-\lambda_{1}(t+\tau)}$ is the leading one in the $\tau \rightarrow \infty$. The second term $e^{-\lambda_{2}(t+\tau)}$ vanishes more quickly, but the subleading contribution determines the critical exponent as we will show later.

In fact, in discussing the subleading contribution, we must also consider the nonlinear effects which were ignored in Eq. (34). We introduce the scaling of $y$ by the replacement $y$ $\rightarrow \alpha y$. We are interested in the limit in which the original $y$ is small, namely, $\alpha \rightarrow 0$. The equation of motion now reads

$$
\frac{d^{2} y}{d t^{2}}=-n \frac{d y}{d t}-\frac{1}{\alpha} \sin (2 \alpha y) .
$$

Considering the limit $\alpha \rightarrow 0$ and retaining only the leading nonlinear term, we obtain

$$
\frac{d^{2} y}{d t^{2}}=-n \frac{d y}{d t}-2 y+\frac{4}{3} \alpha^{2} y^{3}+O\left(\alpha^{3}\right) .
$$

We consider a series expansion of the solution $y$ in terms of $\alpha$, which can be regarded as a perturbative expansion of nonlinear effects.

The lowest order $y^{(0)}$ is given by the solution of linearized equation (34). The next order $y^{(1)}$ is of $O\left(\alpha^{2}\right)$, and is given by a solution of

$$
\frac{d^{2} y^{(1)}}{d t^{2}}+n \frac{d y^{(1)}}{d t}+2 y^{(1)}=\frac{4}{3} \alpha^{2}\left(y^{(0)}\right)^{3} .
$$

This is an inhomogeneous linear differential equation on $y^{(1)}$ for a given $y^{(0)}$, which can be solved by a standard method. Taking the $y^{(0)}$ as general solution (35) of the linear equation, we find the special solution

$$
y^{(1)}(t)=-\alpha^{2} \frac{2 A_{1}^{3}}{3 \lambda_{1}\left(\lambda_{2}-3 \lambda_{1}\right)} e^{-3 \lambda_{1}(t+\tau)}+\cdots,
$$

where only the leading term is given. The general solution also contains solutions of the corresponding homogeneous

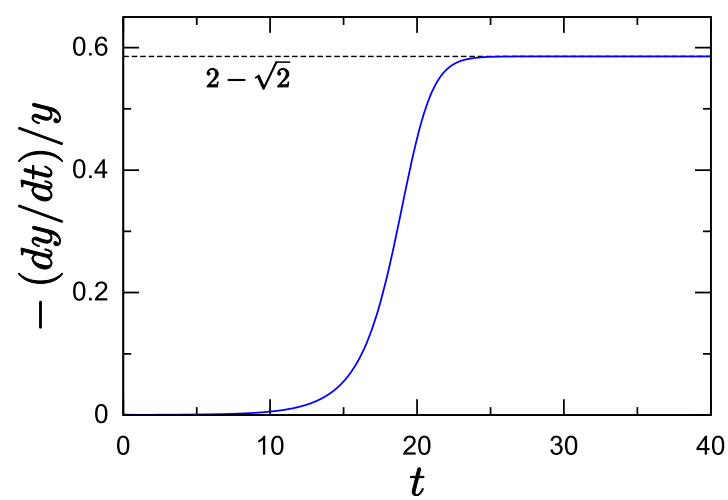

FIG. 1. (Color online) The ratio $-(d y / d t) / y$ as a function of time $t$, within constant- $\eta$ equation (33) and with boundary conditions (26). In the actual numerical calculation, a small initial velocity is given instead of zero in Eq. (26) to avoid getting only the trivial solution. The result is robust against the change in the small initial velocity. In the figure, we show the result for $\eta=4$ as example. The ratio $-(d y / d t) / y$ increases monotonically, asymptotically approaching $\lambda_{1}=2-\sqrt{2} \sim 0.585786$. We obtained similar results for other $\eta>2 \sqrt{2}$.

equation. However, they have the same form as $y^{(0)}$ and can be ignored in the following.

Combining with the solution of the linearized version [Eq. (35)], the leading and next-leading terms in the $\tau \rightarrow \infty$ limit can be written as

$$
y(t)=A_{1} e^{-\lambda_{1}(t+\tau)}\left(1+C e^{-\Delta \lambda(t+\tau)}\right),
$$

where

$$
\Delta \lambda \equiv \min \left(\lambda_{2}-\lambda_{1}, 2 \lambda_{1}\right) .
$$

Namely, when $\lambda_{2}>3 \lambda_{1}$, the next-leading term comes from the nonlinear effect instead of the term proportional to $e^{-\lambda_{2}(t+\tau)}$.

Recalling that the particle approaches $y=0$ from $y=-\pi / 2, A_{1}<0$. The constant $C$ is determined by the solution of nonlinear equation of motion (33) with the constant dissipation and boundary conditions (26). The solution gives the effective initial conditions for the linearized equation.

Numerically solving Eq. (33) with Eq. (26), we find that the ratio $-(d y / d t) / y$ increases monotonically, as shown in Fig. 1, when $n>2 \sqrt{2}$. This observation implies that $C<0$ in linear regime (42).

In fact, when the nonlinear effect gives the next-leading term, it follows from Eq. (41) that

$$
C=-\alpha^{2} \frac{2 A_{1}^{2}}{3 \lambda_{1}\left(\lambda_{2}-3 \lambda_{1}\right)}<0 .
$$

When the subleading contribution within the linear equation gives the next-leading term, we do not have a proof but $C$ $<0$ seems certain from numerical results.

Furthermore, the numerical results indicate that $C$ is sufficiently small so that $y$ never reaches 0 , when asymptotic expression (42) is valid. This, of course, does not mean that there is no solution satisfying $y(0)=0$ in the original Eq. (25), in which the dissipation is turned off around $t \sim 0$. 


\section{Critical parameters in the step-function case}

Let us consider the step-function case of Eq. (30). Here, the solution of Eq. (33) discussed in Sec. III B gives the "initial condition" at $t=-1$ for the equation without the dissipation. If the particle is very close to the minimum $(|y|$ $\ll 1)$ at $t=-1$, the successive motion is just a harmonic oscillation with the angular frequency of $\sqrt{2}$. We will show later that for $n \sim n_{c},|y| \ll 1$ indeed holds at $t=-1$.

The phase $\zeta$ of the oscillation is given as

$$
\tan \zeta=y \sqrt{2}\left(\frac{d y}{d t}\right)^{-1} \text {. }
$$

When $n \rightarrow n_{c}$, the particle just manages to reach $y=0$ at $t$ $=0$. For that, we need to give the optimal initial condition at $t=-1$, namely, $-(d y / d t) / y$ with the maximum possible value. As discussed in Sec. III B, $-(d y / d t) / y$ for Eq. (33) monotonically increases. Thus the optimal initial condition [maximum possible $-(d y / d t) / y]$ is realized by letting the particle spend infinite time around $y \sim 0$ before $t=-1$, namely, by taking $\tau \rightarrow \infty$. In this limit, the first term in Eq. (42) dominates and the initial condition at $t=-1$ is given by

$$
\tan \zeta_{0}=-\frac{\sqrt{2}}{\lambda_{1}}
$$

We emphasize that $T$ depends only on the ratio between the "initial" velocity and "initial" coordinate on $t=-1$, which converges to a finite value in the limit $\tau \rightarrow \infty$. The time required to reach the minimum $(y=0)$ in the harmonic oscillation is

$$
T=\frac{\left|\zeta_{0}\right|}{\sqrt{2}}=\frac{1}{\sqrt{2}} \tan ^{-1} \frac{\sqrt{2}}{\lambda_{1}} .
$$

The critical dissipation coefficient $n_{c}$ in this problem is thus given by

$$
T=\frac{1}{\sqrt{2}} \tan ^{-1} \frac{\sqrt{2}}{\lambda_{1}}=1,
$$

so that the particle arrives at $y=0$ at $t=0$. Therefore we find

$$
n_{c}=\frac{2 \sqrt{2}}{\sin 2 \sqrt{2}} \sim 9.18107 \cdots .
$$

In the limit $n \rightarrow n_{c}, \tau \rightarrow \infty$, and thus both $y(-1)$ and $d y / d t$ $(-1)$ vanish. This implies that the velocity of the particle when it reaches the potential minimum, $d y / d t(0)$, also vanishes. Let us discuss its critical behavior, namely, how $d y / d t(0)$ depends on $n_{c}-n$ when $n \leqq n_{c}$.

For $n<n_{c}$, if we take $\tau \rightarrow \infty$ the particle reaches the minimum before $t=0$ because $T<1$. By letting the particle spend less time around $y \sim 0$ by taking a smaller $\tau$, we can change the initial condition at $t=-1$ so that $\zeta_{0}$ is smaller than the optimal value (46). Therefore, for $n<n_{c}$, there is a solution which reaches the minimum at $t=0$. Combining

$$
-\frac{1}{\sqrt{2} y}\left(\frac{d y}{d t}\right)(t=-1)=\tan \frac{1}{\sqrt{2}}
$$

with Eq. (42), for small $n_{c}-n$ we find

$$
\left.\frac{\partial \lambda_{1}}{\partial n}\right|_{n=n_{c}}\left(n-n_{c}\right)+\left(\lambda_{2}-\lambda_{1}\right) C e^{-\Delta \lambda(\tau-1)} \sim 0 .
$$

This implies that

$$
e^{-\Delta \lambda \tau} \propto n_{c}-n
$$

As a consequence,

$$
y(-1) \propto \frac{d y}{d t}(-1) \propto e^{-\lambda_{1} \tau} \propto\left(n_{c}-n\right)^{\lambda_{1} / \Delta \lambda},
$$

which leads to

$$
\frac{d y}{d t}(0) \propto\left(n_{c}-n\right)^{\lambda_{1} / \Delta \lambda} .
$$

In the present case, for $n \sim n_{c}, \lambda_{2}>3 \lambda_{1}$, namely, $\Delta \lambda=2 \lambda_{1}$. Thus we obtain

$$
\frac{d y}{d t}(0) \propto\left(n_{c}-n\right)^{1 / 2} .
$$

For $n>n_{c}$, even under the optimal condition $\tau \rightarrow \infty$, the particle cannot reach $y=0$ at $t=0$. In this case, the odd solution does not exist.

\section{Critical parameters in the tanh case}

Now let us consider tanh case (31). The mathematics is somewhat more complicated but the physics is quite similar to the previous one.

As in the previous problem, for $n \sim n_{c}$, we can assume that the particle comes very close to the minimum $y \sim 0$ at negative time. The linearized equation of motion, with the full time dependence of the dissipation, reads

$$
\frac{d^{2} y}{d t^{2}}=+n \frac{d y}{d t} \tanh t-2 y \text {. }
$$

This equation has the general solution

$$
\begin{aligned}
y= & C_{1} P_{n / 2}^{\left(\sqrt{\left.n^{2}-8 / 2\right)}\right.}(\tanh t)\left(1-\tanh ^{2} t\right)^{-n / 4} \\
& +C_{2} Q_{n / 2}^{\left(\sqrt{\left.n^{2}-8 / 2\right)}\right.}(\tanh t)\left(1-\tanh ^{2} t\right)^{-n / 4},
\end{aligned}
$$

where $C_{1,2}$ are constants, and $P_{\mu}^{(\nu)}$ and $Q_{\mu}^{(\nu)}$ are associated Legendre functions.

Let us first discuss the asymptotic behavior of the above solution in the limit $t \rightarrow-\infty$. In this limit, equation of motion (56) reduces to Eq. (34), and thus the solution should be equivalent to Eq. (35). In fact, using

$$
\tanh t \sim-1+2 e^{2 t}+O\left(e^{4 t}\right)
$$

and the asymptotic expansion of the associated Legendre functions, we have confirmed that Eq. (57) coincides with Eq. (35) in the limit $t \rightarrow-\infty$.

Now, the similar discussion as in Sec. III C applies here. Namely, the numerical solution implies that $-(d y / d t) / y$ increases monotonically. Thus, the optimal condition for reaching $y=0$ is realized when the particle spends infinite time around $y \sim 0$ before $t \sim 0$. This can be done by replacing $t$ with $t+\tau$ and taking $\tau \rightarrow \infty$. In this limit, the asymptotic be- 


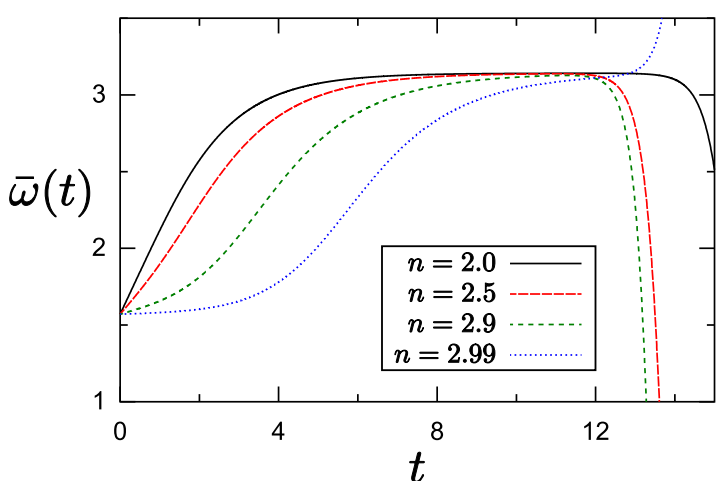

FIG. 2. (Color online) Trajectories of $\bar{\omega}(t)$ in the case of $\eta(t)$ $=-n \tanh t$. The trajectories in $t<0$ are antisymmetric to that in $t$ $>0$, so we show $\bar{\omega}(t)$ only for $t>0$. Here, calculating the trajectories numerically, $\bar{\omega}(t=0)=\pi / 2$ is imposed as an initial condition. Now, although we define $n>2$, the trajectory for $n=2$ is also shown.

havior of the solution in the $t \ll-1$ regime is dominated by $e^{-\lambda_{1}(t+\tau)}$.

In the following, we demonstrate that

$$
n_{c}=3 \text {. }
$$

To show that, let us set $n=3$. The two independent solutions reduce to

$$
\begin{gathered}
P_{3 / 2}^{(1 / 2)}(\tanh t)\left(1-\tanh ^{2} t\right)^{-3 / 4} \sim \frac{1}{2 \sqrt{2 \pi}} e^{-\lambda_{2} t}, \\
Q_{3 / 2}^{(1 / 2)}(\tanh t)\left(1-\tanh ^{2} t\right)^{-3 / 4} \sim \sqrt{\frac{\pi}{2}} e^{-\lambda_{1} t}
\end{gathered}
$$

in the limit $t \rightarrow-\infty$. This implies that under the optimal condition, the solution consists only of the $Q_{3 / 2}^{(1 / 2)}$ term.

On the other hand, for $n=3$, the Taylor expansion around $t=0$ reads

$$
\begin{aligned}
& P_{3 / 2}^{(1 / 2)}(\tanh t)\left(1-\tanh ^{2} t\right)^{-3 / 4} \sim-\sqrt{\frac{2}{\pi}}+O\left(t^{2}\right), \\
& Q_{3 / 2}^{(1 / 2)}(\tanh t)\left(1-\tanh ^{2} t\right)^{-3 / 4} \sim-\sqrt{2 \pi} t+O\left(t^{2}\right) .
\end{aligned}
$$

Namely, the solution consisting only of the $Q$ term just crosses $y=0$ on $t=0$. By perturbing the solutions around $n$ $=3$, it can be shown that the solution with $y(0)=0$ exists for $n<3$. This means that $n=3$ is indeed the critical value $n_{c}$.

As in the case in Sec. III C, the velocity at the potential minimum $d y / d t(0)$ vanishes as $n$ approaches $n_{c}$ from below. The critical behavior can be obtained by a similar argument, and is given by Eq. (54). In the present case, $\lambda_{1}=1$ and $\lambda_{2}$ $=2<3 \lambda_{1}$. Thus we find the linear behavior

$$
\frac{d y}{d t}(0) \propto\left(n_{c}-n\right)
$$

when $n \lesssim n_{c}$.

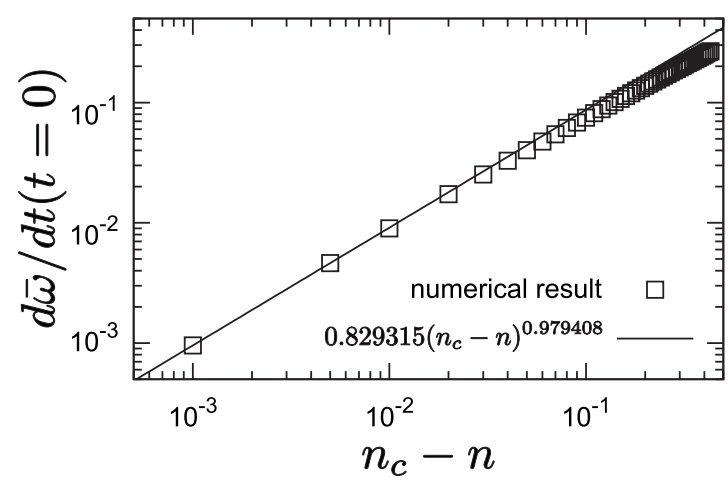

FIG. 3. The velocity of the particle at $t=0$ as a function of $n_{c}$ $-n$, for the tanh dissipation [Eq. (31)]. The squares are numerical results obtained by the shooting method. The line is the best fit assuming a power law. The critical parameter $n_{c}$ is determined by the analytic prediction. The good fit to the power law means that the analytic prediction of $n_{c}$ is consistent with the numerical calculation. Moreover, the exponent $0.979 \cdots$ obtained by the fit is also consistent with the analytic prediction of unity .

\section{E. Requirement of a finite number of bosons}

We consider the situation where all the bosons are confined in the finite space by external trap potential $V(\boldsymbol{r})$. If it is to be realized in experiments, the total number of the bosons should be finite. This gives an additional requirement independent of the stability.

In terms of the density profile $f_{0}^{2}$, the condition of the finite number of bosons is easily given as

$$
\int d \widetilde{\boldsymbol{r}} f_{0}^{2}(\widetilde{r})=4 \pi \int_{0}^{\infty} d \widetilde{r}^{2} f_{0}^{2}(\widetilde{r})<\infty .
$$

For the choice of the tanh dissipation [Eq. (31)], the density profile can be obtained via Eq. (19) as

$$
f_{0}^{2}(\widetilde{r})=B \frac{\widetilde{r}^{n-1}}{\left(1+\widetilde{r}^{2}\right)^{n}},
$$

where $B$ is a positive constant which appears from the integration of Eq. (19). The density profile in the $n=1$ case was discussed in Ref. [13]. $\widetilde{r}^{2} f_{0}^{2}(\widetilde{r})$ in the integral of Eq. (65) asymptotically behaves as $\sim \widetilde{r}^{-n+1}$ for large $\widetilde{r}$. Accordingly, for $n \leq 2$ the total number of the trapped bosons diverges, violating condition (65). Hereafter, we focus on the finiteboson case, $n>2$.

\section{NUMERICAL SOLUTION FOR A SKYRMION}

\section{A. Solution of the equation of motion}

Equation of motion (17) determines $\bar{\omega}(t)$ for a given choice of $\eta(t)$. While we have determined critical parameters in Sec. III, unfortunately, the full solution cannot be obtained analytically. Thus, here we solve Eq. (17) numerically. The numerical solution can be also used to check the analytical predictions on the critical parameters discussed in Sec. III.

To reiterate, we seek a solution which satisfies the boundary conditions at $t= \pm \infty$ [Eq. (20)]. For an odd $\eta(t)$, which is the case we discuss in this paper, such a solution satisfies Eq. 


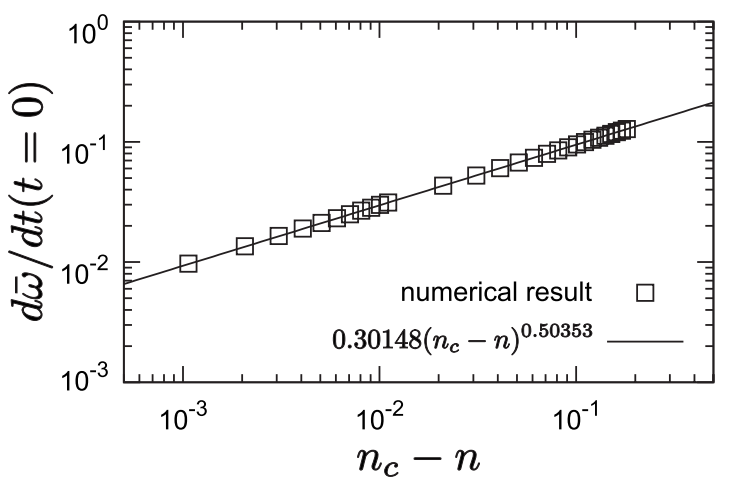

FIG. 4. The velocity of the particle at the potential minimum $(t=0)$ as a function of $n_{c}-n$, for the step-function dissipation. The line is the best fit assuming a power law to the numerical results, which are shown as squares. The critical parameter $n_{c}$ is determined by the analytic prediction. The numerical result is again in good agreement with the predictions.

(28). To find the odd solution for an odd $\eta(t)$, it is enough to require

$$
\bar{\omega}(0)=0,
$$

together with either of the boundary conditions at $t=-\infty$ or $t=\infty$ in Eq. (20).

Based on this observation, we adopt the so-called shooting method in the numerical scheme, which is explained in Appendix A. In Fig. 2, we show the numerical result for the case of Eq. (31).

Here, we observe that the velocity at $t=0$ vanishes as the dissipation parameter $n$ approaches $n_{c}=3$. This is indeed consistent with the analytic prediction on the critical parameter $n_{c}=3$ and on the critical behavior. To see this more clearly, in Fig. 3, we show the numerical result on $d \bar{\omega} / d t(0)$ $=d y / d t(0)$ as a function of $n_{c}-n$. The result is in good agreement with the analytic predictions. We also have made a similar comparison for step-function dissipation (30) in Fig. 4 , and found agreement with analytic predictions (49) and (55) as well.

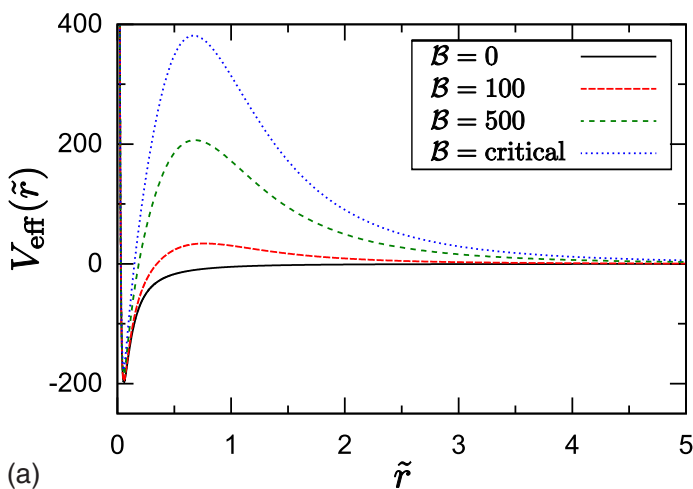

FIG. 5. (Color online) Plots of the effective potential $V_{\text {eff }}$ defined in Eq. (23) for given $\eta(t)=-n \tanh t$. In the left graph (a), $V_{\text {eff }}(\widetilde{r})$ with fixed $n=2.5$ are plotted. In the right graph (b), the $n$ dependence of $V_{\text {eff }}(\widetilde{r})$ with fixed $\mathcal{B}=0$ is shown. For each value of $n$, there is a critical value of $\mathcal{B}$ which makes the ground-state energy of Hamiltonian (22) exactly zero. In the region of $\mathcal{B}$ shown in the left graph (a), the ground-state eigenvalues are negative.

\section{B. Stability of the skyrmion and minimal number of bosons}

We have shown that the skyrmion as a solution of the Euler-Lagrange equation exists for $n<n_{c}$, and obtained the solution numerically. The spinor configuration $\bar{\omega}(t)$ is given by the solution of a classical mechanics problem. The density profile $f_{0}(\widetilde{r})$ can then be obtained.

The next step is to examine the stability of the obtained skyrmion. Note that there is a free parameter $B$ appearing in density profile (66). Substituting the obtained $\bar{\omega}(t)$ and $\bar{f}_{0}(t)$ into Euler-Lagrange equation (16), the trap potential $\tilde{V}(\widetilde{r})$ realizing the skyrmion is obtained. Then, we find that $\tilde{V}(\widetilde{r})$ depends on $\tilde{U}$ and $B$ as a function of $\tilde{U} B=\mathcal{B}$. This information on $\omega(\widetilde{r}), f_{0}(\widetilde{r})$, and $\widetilde{V}(\widetilde{r})$ determines effective potential (23), shown in Fig. 5.

In order to obtain the stable skyrmion, $\mathcal{B}$ larger than the critical value $\mathcal{B}_{\mathrm{c}}$ is needed. Indeed, calculating the groundstate energy of quantum-mechanical Hamiltonian (22) by use of the numerical diagonalization method, it is found that as $n$ increases, the critical value $\mathcal{B}_{\mathrm{c}}$ diverges as $n$ approaches $n_{c}$ $=3$, as shown in Fig. 6 .

Finally, let us investigate the total number of the trapped bosons for some values of $\mathcal{B}$ and $n$. Due to Eq. (66) for the density profile, the number of bosons monotonically increases as a function of $\mathcal{B}$ : it is proportional to $\mathcal{B}$. For a given $n$, the number of the trapped bosons is minimal at $\mathcal{B}=\mathcal{B}_{\mathrm{c}}$. The $n$ dependence of the number of the bosons in $\mathcal{B}=\mathcal{B}_{\mathrm{c}}$ is then shown in Fig. 7. We find the minimum at $n \sim 2.25$, with the value of $\sim 10^{4}$. It is comparable to the typical numbers in the experiments.

\section{SUMMARY}

In this paper, we have discussed the skyrmion configurations of the two-component spinor BECs confined by a trap potential. The necessary condition for the existence of the skyrmion has been formulated earlier by two of the authors [13]. The Euler-Lagrange equation, which must be satisfied by the skyrmion, turned out to give an equation of motion for a fictitious classical particle subject to time-dependent dissipation. A skyrmion solution satisfying appropriate boundary

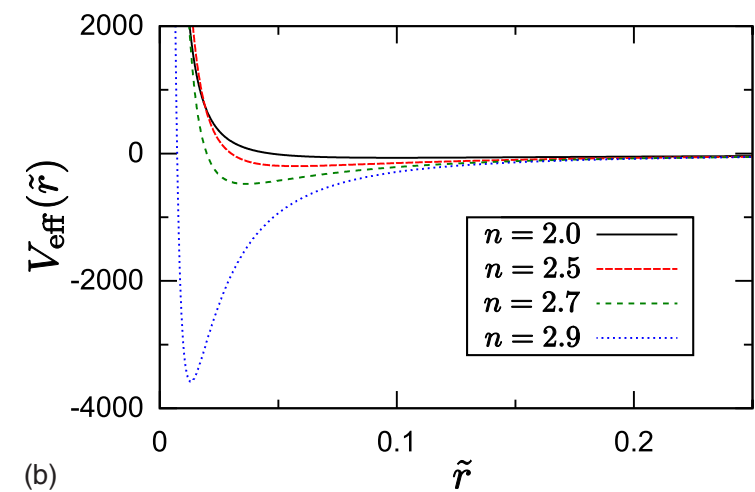




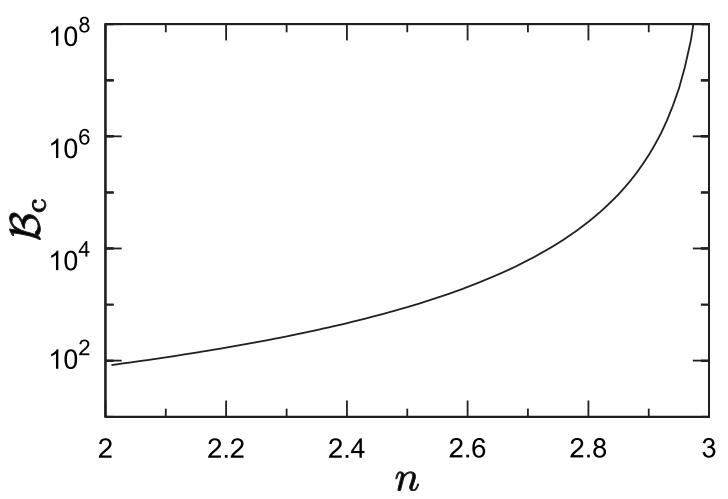

FIG. 6. The critical value $\mathcal{B}_{\mathrm{c}}$ as a function of $n$. The values of $\mathcal{B}_{\mathrm{c}}$ are plotted on logarithmic scale. We find that $\mathcal{B}_{\mathrm{c}}$ increases with $n$. Eventually, in the limit $n \rightarrow 3, \mathcal{B}_{\mathrm{c}}$ diverges.

conditions exists only for specially chosen trap potentials. Some of those trap potentials were constructed by considering dissipation which is an odd function of time. It was expected to allow a solution of the equation of motion, which is odd in time.

In this paper, we have developed this approach further. We have mainly considered two classes of solutions, obtained by taking the dissipation as proportional to the step function of time [Eq. (30)], and to hyperbolic tangent of time [Eq. (31)]. We have found that, even though these choices of dissipation are odd in time, they do not allow a skyrmion solution if the dissipation is too strong. We have obtained the critical parameter exactly, and also determined a critical behavior in the velocity at the potential minimum. These predictions are verified by numerical solution.

Furthermore, we discussed the stability of the skyrmion, taking into account the second-order variational theory. In our formulation the problem of the stability of the skyrmion is mapped onto that of the sign of the lowest eigenvalue of a certain quantum-mechanical Hamiltonian determined by the skyrmion solution.

For the tanh model, the density profile and the trap potential reproducing the skyrmion have several parameters $\tilde{U}, n$, and $B$, and we determined the region in the parameter space that leads to a stable skyrmion. For a given $n, \mathcal{B}=\widetilde{U} B$ needs

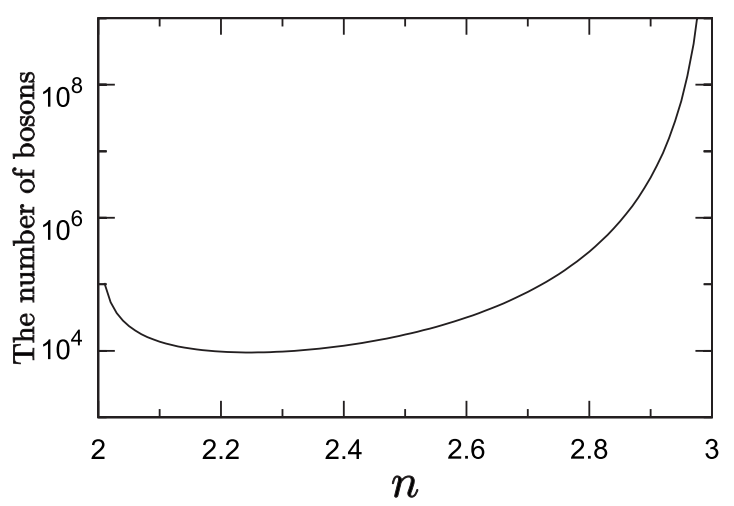

FIG. 7. The $n$ dependence of the number of the trapped bosons in $B=\widetilde{U}^{-1} \mathcal{B}_{\mathrm{c}}$ for a given $n$. It has the minimal value of $\sim 10^{4}$ around $n=2.25$.

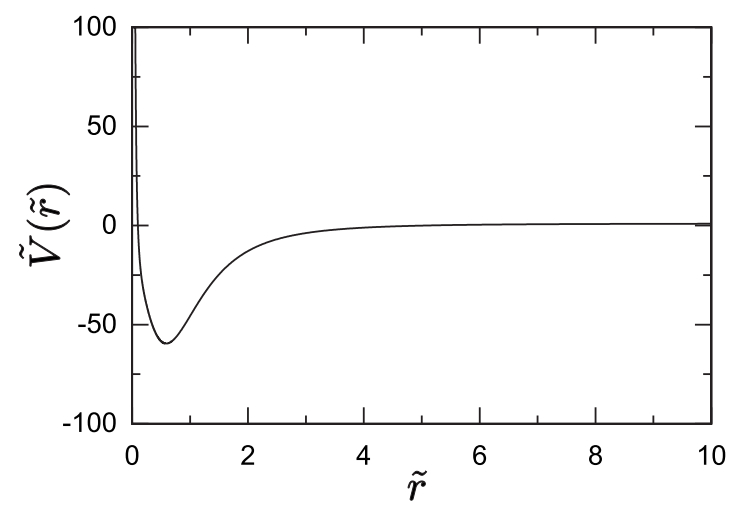

FIG. 8. The example of the trap potential $\tilde{V}(\widetilde{r})$ reproducing the skyrmion. The density profile under this potential is given by Eq. (66) with $n=2.25$ and $\mathcal{B}=213.495$. As seen in Fig. 7, for these $n$ and $\mathcal{B}$, the number of the trapped bosons is minimal $\left(\sim 10^{4}\right)$.

to be larger than a certain value for the skyrmion to be stable (see Fig. 6).

We have found that there exists a minimal number of bosons of $\sim 10^{4}$ allowing a stable skyrmion solution. For illustration, the trap potential required to reproduce the skyrmion with this minimal number of particles is shown in Fig. 8. In order to connect our results to real experiments, it would be important to see the asymptotic form of the trap potential stabilizing the skyrmion. It can be analytically derived from asymptotic analysis of differential equations (15) and (16). In the generic case that the odd $\eta(t)$ asymptotically reaching $\mp n$, respectively, as $t \rightarrow \pm \infty$, as seen in Appendix $\mathrm{B}$, the form of the trap potential can be obtained as Eqs. (B5) and (B6), respectively, for $\tilde{r} \ll 1$ and $\tilde{r} \gg 1$. One can easily find that these equations are consistent with the result of the analytically solvable cases discussed in Ref. [13]: $\eta(t)=0$ for $n=0$ and $\eta(t)=-\tanh t$ for $n=1$. For $n>2$, the leading terms in Eqs. (B5) and (B6) are both proportional to $\sim 1 / \widetilde{r}^{2}$. Interestingly, the power of the leading terms is independent of $n$. In particular, in the $n=2.25$ case shown in Fig. 8, the analytically obtained asymptotic form for $\widetilde{r} \ll 1,1 / \widetilde{r}^{2}$, is in good agreement with the numerical result. On the other hand, in the case of the large- $\widetilde{r}$ side, because of the very large prefactor $\mathcal{B} \sim 10^{2}$, the subleading term remains effective. Thus, taking into account the leading term as well as the subleading term $\sim-\mathcal{B} / \widetilde{r}^{n+1}$, the analytical result for $n=2.25$ matches the numerical one very well.

There are many open problems which would deserve further study. In this paper we only discussed skyrmions in equilibrium. Dynamical aspects, such as relaxation to the skyrmion solution, are also important. The dynamical aspects would be even more crucial in possible physical realizations of our proposal. As we have shown, the potential has to be fine-tuned to allow a stable skyrmion solution. In reality, it is impossible to construct a potential with infinite precision and thus there would be some error in the potential. This would make skyrmions absent, as a stable solution in equilibrium. However, we expect that if the actual potential is close enough to the exact one with a skyrmion solution, the skyrmion would exist as a quasistable state with a certain lifetime. In order to discuss the feasibility of observation of a 
skyrmion, we would need to estimate the lifetime of the skyrmion in the actual potential. We hope progress will be made on these problems, and also on other directions related to our study.

\section{ACKNOWLEDGMENTS}

This work was supported in part by 21st Century COE programs at Tokyo Institute of Technology, "NanometerScale Quantum Physics," and at Hokkaido University, "Topological Science and Technology," and Grant-in-Aid for Exploratory Research No. 20654030, from MEXT, Japan. A.T. was supported by JSPS. I.F.H. was supported by the NSERC of Canada.

\section{APPENDIX A: SHOOTING METHOD}

In general, iterating the discretized time step based on the boundary condition, the equation of motion can be numerically solved. Based on this observation, we adopt the socalled shooting method in the numerical scheme as follows:

(1) Choose the initial velocity $d \bar{\omega} / d t(0)$ arbitrarily.

(2) Given the initial velocity and the initial position $\bar{\omega}(0)=0$, solve equation of motion (17) toward $t=\infty$.

(3) If the particle goes beyond the peak of the potential at $\bar{\omega}=\pi$, the initial velocity was too large.

(4) If the particle comes back without reaching the peak of the potential at $\bar{\omega}=\pi$, the initial velocity was too small.

In a true solution, the particle should approach asymptotically the peak of the potential $\bar{\omega}=\pi$ as $t \rightarrow \infty$. However, the asymptotic behavior in $t \rightarrow \infty$ is quite sensitive to the initial velocity, and the numerical solution departs from the peak of the potential in either way, depending on the tiny difference of order of machine precision, in the initial velocity. The sensitivity is due to the instability of the particle at the potential peak, further enhanced by the negative dissipation coefficient. On the other hand, because of this sensitivity, we can easily obtain the correct initial velocity in a high precision.

In practice, the shooting method can be implemented as an efficient iteration using the bisection method as follows:

(1) Find the two values of the initial velocity $\left\{v_{1}^{L}, v_{1}^{U}\right\}$, so that $v_{1}^{L}$ is "too small" and $v_{1}^{U}$ is "too large."

(2) On $n$th iteration, the correct initial velocity should be within the range $\left(v_{n}^{L}, v_{n}^{U}\right)$. Thus, numerically solve the equation of motion with the midpoint initial velocity $v_{n}^{M}$ $\equiv\left(v_{n}^{L}+v_{n}^{U}\right) / 2$.

(3) If the midpoint $v_{n}^{M}$ is too small as the initial velocity, set $v_{n+1}^{L}=v_{n}^{M}, v_{n+1}^{U}=v_{n}^{U}$. If $v_{n}^{M}$ is too large, set instead $v_{n+1}^{L}$ $=v_{n}^{L}, v_{n+1}^{U}=v_{n}^{M}$. Go to step 2 as the $(n+1)$ th iteration.

In this way, the error in the initial velocity decreases proportionally to $2^{-n}$ in the $n$th iteration.

\section{APPENDIX B: ASYMPTOTIC FORM OF TRAP POTENTIALS}

In order to drive the asymptotic form of trap potentials, we rewrite equation of motion (17) for $t \ll-1$ and $t \gg 1$. Then, in this odd-dissipation case $\eta(t)$ can be approximated as $\eta(t) \approx n$ for $t \ll-1$ and $\eta(t) \approx-n$ for $t \gg 1$. In addition, as shown by the numerical results, the solution would stand near $\bar{\omega}(t \ll-1) \approx 0$ and $\bar{\omega}(t \gg 1) \approx \pi$. From these assumptions, equation of motion (17) is linearized as

$$
\frac{d^{2} \bar{\omega}}{d t^{2}}=2 \bar{\omega}-n \frac{d \bar{\omega}}{d t}
$$

for $t \ll-1$, and

$$
\frac{d^{2} \bar{\omega}}{d t^{2}}=-2 \pi+2 \bar{\omega}+n \frac{d \bar{\omega}}{d t}
$$

for $t \gg 1$. The solutions satisfying the boundary conditions, $\lim _{t \rightarrow-\infty} \bar{\omega}(t)=0$ and $\lim _{t \rightarrow+\infty} \bar{\omega}(t)=\pi$, can be easily obtained, respectively, as

$$
\bar{\omega}(t)= \begin{cases}D_{1} e^{\lambda t} & (t \ll-1) \\ \pi+D_{2} e^{-\lambda t} & (t \gg 1),\end{cases}
$$

where $\lambda=\frac{-n+\sqrt{n^{2}+8}}{2}>0 . D_{1}$ and $D_{2}$ are unknown constants. On the other hand, from the boundary conditions for $\eta(t)$ and the definition of the dissipation $\eta(t)=1+\frac{d}{d t} \ln \vec{f}_{0}^{2}$, the asymptotic forms of the density profile for $t \ll-1$ and $t \gg 1$ are easily obtained as

$$
\bar{f}^{2}(t) \propto \begin{cases}e^{(n-1) t} & (t \ll-1) \\ e^{-(n+1) t} & (t \gg 1) .\end{cases}
$$

Taking asymptotic forms (B3) and (B4) from the $t$ representation to $\tilde{r}$ representation by $t=\ln \tilde{r}$, and substituting them into one of equations of motion (15), the trap potential $\tilde{V}(\widetilde{r})$ can be derived. As a result, $\tilde{V}(\widetilde{r})$ for $\widetilde{r} \ll 1$ and $\tilde{r} \gg 1$ are investigated as

$$
\tilde{V}(\tilde{r})=1-D_{1}^{2} \frac{\lambda^{2}+2}{2 \widetilde{r}^{2-2 \lambda}}+\frac{n^{2}-1}{8 \widetilde{r}^{2}}-B_{1} \frac{\tilde{U}}{\tilde{r}^{1-n}}
$$

for $\tilde{r} \ll 1$, and

$$
\tilde{V}(\widetilde{r})=1-D_{2}^{2} \frac{\lambda^{2}+2}{2 \widetilde{r}^{2+2 \lambda}}+\frac{n^{2}-1}{8 \widetilde{r}^{2}}-B_{2} \frac{\tilde{U}}{\tilde{r}^{1+n}}
$$

for $\tilde{r} \gg 1$. $B_{1}$ and $B_{2}$ are unknown constants and are the prefactors of the density profile, respectively, for $t \ll-1$ and $t$ $\gg 1$. In particular, in the case of $\eta(t)=-n$ tanh $t$ discussed in this paper, they turn out to be $B$ appearing in Eq. (66): $B_{1}$ $=B_{2}=B$. The leading term is decided by the value of $n$. However, in Fig. 8, because of the very large prefactor $B \tilde{U}$ $\sim 10^{2}$, the subleading term still remains effective in shown region of $\tilde{r}$. 
[1] I. Herbut, A Modern Approach to Critical Phenomena (Cambridge University Press, Cambridge, England, 2007).

[2] A. A. Belavin and A. M. Polyakov, JETP Lett. 22, 245 (1975).

[3] N. Manton and P. Sutcliffe, Topological Solitons (Cambridge University Press, Cambridge, England, 2004).

[4] M. H. Lau and C. Dasgupta, Phys. Rev. B 39, 7212 (1989); M. Kamal and G. Murthy, Phys. Rev. Lett. 71, 1911 (1993); N. D. Antunes, L. M. A. Bettencourt, and M. Kunz, Phys. Rev. E 65, 066117 (2002); O. I. Motrunich and A. Vishwanath, Phys. Rev. B 70, 075104 (2004).

[5] E. Eisenberg and E. H. Lieb, Phys. Rev. Lett. 89, 220403 (2002).

[6] A. Vilenkin and A. P. S. Shellard, Cosmic Strings and Other Topological Defects (Cambridge University Press, Cambridge, England, 1994).

[7] For a recent review, see K. Kasamatsu, M. Tsubota, and M. Ueda, Int. J. Mod. Phys. B 19, 1835 (2005).
[8] Y. Saiga and M. Oshikawa, Phys. Rev. Lett. 96, 036406 (2006).

[9] I. F. Herbut, Phys. Rev. Lett. 94, 237001 (2005); 88, 047006 (2002); Phys. Rev. B 66, 094504 (2002).

[10] M. Franz, Z. Tešanović, and O. Vafek, Phys. Rev. B 66, 054535 (2002); Z. Tešanović, Phys. Rev. Lett. 93, 217004 (2004).

[11] T. Senthil and M. P. A. Fisher, Phys. Rev. B 74, 064405 (2006).

[12] G. H. Derrick, J. Math. Phys. 5, 1252 (1964).

[13] I. F. Herbut and M. Oshikawa, Phys. Rev. Lett. 97, 080403 (2006).

[14] See also L. Lichtensteiger and R. Durrer, Phys. Rev. D 59, 125007 (1999).

[15] L. D. Landau and E. M. Lifshitz, Mechanics (Pergamon, Oxford, 1976), Chap. 5. 